\title{
Role of pharmacists in optimizing the use of anticancer drugs in the clinical setting
}

This article was published in the following Dove Press journal:

Integrated Pharmacy Research and Practice

19 February 2014

Number of times this article has been viewed

\section{Carolyn SJ Ma}

Department of Pharmacy Practice, Daniel K. Inouye College of Pharmacy, University of Hawaii at Hilo, Honolulu, $\mathrm{HI}$, USA
Correspondence: Carolyn SJ Ma Department of Pharmacy Practice, Daniel K Inouye College of Pharmacy, University of Hawaii at Hilo, 677 Ala Moana Blvd. Suite 1025, Honolulu, $\mathrm{HI} 96813$, USA

Tel + I 8085872697

Email csjma@hawaii.edu
Abstract: Oncology pharmacists, also known as oncology pharmacy specialists (OPSs) have specialized knowledge of anticancer medications and their role in cancer. As essential member of the interdisciplinary team, OPSs optimize the benefits of drug therapy, help to minimize toxicities and work with patients on supportive care issues. The OPSs expanded role as experts in drug therapy extends to seven major key elements of medication management that include: selection, procurement, storage, preparation/dispensing, prescribing/dosing/transcribing, administration and monitoring/evaluation/education. As front line caregivers in hospital, ambulatory care, longterm care facilities, and community specialty pharmacies, the OPS also helps patients in areas of supportive care including nausea and vomiting, hematologic support, nutrition and infection control. This role helps the patient in the recovery phase between treatment cycles and adherence to chemotherapy treatment schedules essential for optimal treatment and outcome.

Keywords: oncology pharmacist, oncology pharmacy specialist, medication management, chemotherapy

\section{Introduction}

Pharmacists began training and defining their role in medical oncology practice in 1976, when Morris and Hickman outlined a curriculum for pharmacy students at the University of Tennessee in the US to help prepare them to participate in the therapeutic care of cancer patients. ${ }^{1}$ The foundation curriculum for pharmacist training differentiates itself from other health care professions with emphasis on pharmacokinetics of drug behavior in the liver and kidneys as well as extensive training in pharmacology and medicinal chemistry. The American Society of Health-System Pharmacists (ASHP) published guidelines in 1990, 1993, 1996, and 2002 to describe a pharmacist's role not only in safe handling, preparation, and dispensing of drugs but also in pharmaceutical care as the health professional who is "... directly responsible for the provision outlined for medication-related care for the purpose of achieving definite outcomes that improve a patient's quality of life. The pharmacist is deemed responsible for identifying, resolving, and preventing medication-related problems such as untreated indications, improper drug selection, inadequate dosing, adverse drug reactions, interactions and medication use without indication." $2-6$

The Board of Pharmacy Specialties, an autonomous division of the American Pharmacists Association, recognizes oncology pharmacy as one of eight specialty areas of pharmacy with the designation of Board Certified Oncology Pharmacists (BCOP). ${ }^{6}$ Other countries have similar specialty designations through their respective pharmacy professional organizations. As of January 2014, 1,635 pharmacists were 
board certified in the US as BCOP. ${ }^{7}$ Oncology pharmacy specialists (OPS) have expanded their role as experts in specific cancers and are front line caregivers in supportive care for the side effects caused by chemotherapy and biological agents. The OPS work on supportive care issues such as nutrition support, pain, infection control, hematology and anticoagulation as described in Table 1. This role helps the patient in the recovery phase between treatment cycles and adherence to chemotherapy treatment schedules essential for optimal treatment and outcome. ${ }^{8}$ Due to the plethora of safety issues with drugs with a low therapeutic index, OPS focus on different aspects of patient care that include their expertise with anti-cancer drugs themselves as well as the management of the numerous side effects caused by these drugs (Table 2).

The American Society of Clinical Oncology describes oncology pharmacists as having specialized knowledge of medications and their role in cancer and essential interdisciplinary team members who maximize the benefits of drug therapy and minimize toxicities. ${ }^{8}$ Figure 1 depicts the range of pharmacists' skills from broader responsibilities to more specialized expertise.

This descriptive review paper focuses on the various components of medication management and the OPS's role from a viewpoint of clinical practice in the US. Each of these processes helps to support optimal anticancer drug treatment. Activities take place in the inpatient hospital, outpatient ambulatory care clinics, specialty community pharmacies, and long-term care centers where OPSs are located. This paper shares information that could be utilized worldwide, given that oncology pharmacy practices

Table I Oncology pharmacist practice for supportive care

- Hematology support

- Anemia EPO, non-EPO

- Colony-stimulating factor

- Anticoagulation clinic

- Chemotherapy administration follow-up

- Chemoprotective agents - protocol development, dosing

- Gastrointestinal side effect support - N/V/D/C/mucositis

- Cutaneous/dermatological support - extravasation, hand-foot syndrome, hand-foot rash syndrome, maculopapular rash management

- Pain management - opiates, side effect management, nonnarcotic medications

- Infectious disease and antibiotic support

- Immunization

- Parenteral nutrition support

- Palliative care

- Chronic disease medication management - hypertension, hyperlipidemia, cholesterol management, asthma control, diabetes control

Notes: Data taken from. ${ }^{10,11,120,121}$

Abbreviations: EPO, erythropoietin; N/VID/C, nausea/vomitting/diarrhea/constipation.
Table 2 Oncology pharmacist drug-specific interventions

- Adverse drug-reaction prevention and monitoring

- Medication administration support, premedication, hydration

- Addition of necessary medications

- Discontinuation of drugs

- Dose adjustments for organ dysfunction, weight, age

- Therapeutic drug monitoring

- Pharmacokinetic monitoring

- Laboratory monitoring

- Switch intravenous to by-mouth formulations

- Writing prescriptions and refills

- Medication reconciliation and allergies

- Patient education

- Assuring medication adherence

Note: Data taken from. ${ }^{10,11,120,121}$

vary greatly. References were resourced via the PubMed search engine and accessed via the University of Hawaii library system.

As a starting point, the Joint Commission, which is the accreditation body for health care facilities in the US and for international institutions who seek US thirdparty insurance reimbursements, outlined a process of seven critical steps that constitute safe and complete medication management: 1) selection; 2) procurement; 3) prescribing, dosing, and transcribing; 4) storage; 5) preparing and dispensing (includes delivery); 6) administering; and 7) monitoring, evaluation, and education. ${ }^{9,10}$ Figure 2 depicts the components of this process.

\section{Medication-management components Selection}

Although selection could be confused with prescribing in some countries, the Joint Commission defines selection in this case as appropriate choice of a medication for a specific indication. ${ }^{9}$ OPSs can aid in the area of appropriate selection and provide medical information about antineoplastic pharmacology, dosing adjustments for organ dysfunction, and adverse-effect profiles. Drug-information skills specific to OPSs include the ability to utilize appropriate search engines and find published clinical trials and ongoing clinical trials, review study design, and evaluate evidence-based guidelines and appropriateness of biostatistics. ${ }^{10}$ Frequently asked questions of pharmacists may include requests to find the latest information on availability of an investigational anticancer drug and find updated information on recent developments. Additional information the OPS may regularly access is literature that documents use of an anticancer drug for off-label use. This information can be particularly helpful to justify possible health insurance reimbursement. OPSs also perform admission medication 


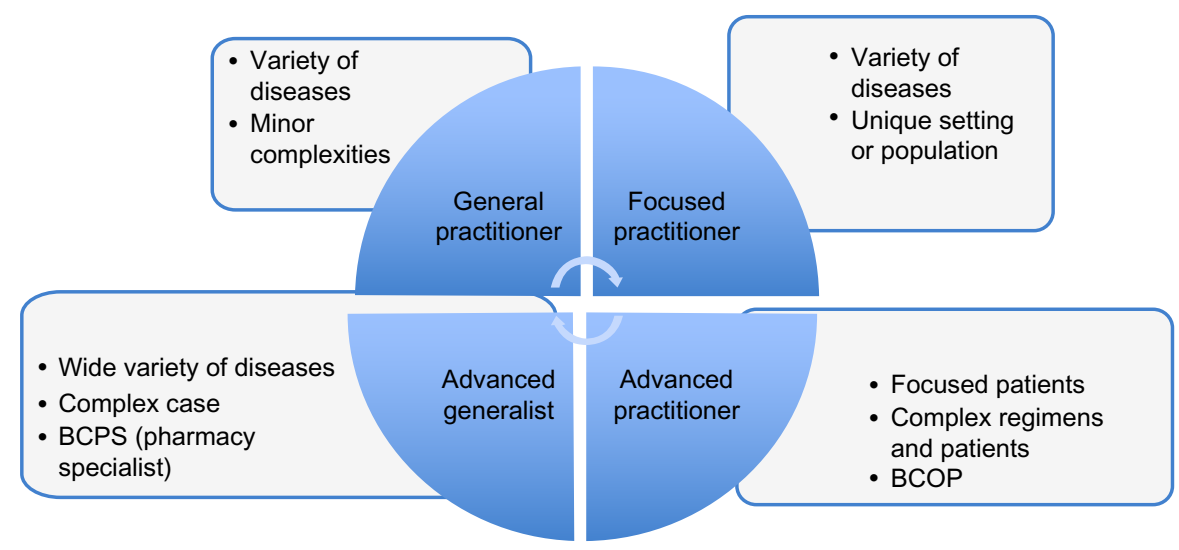

Figure I Range of pharmacist skills.

Notes: Adapted with permission from Council on Credentialing in Pharmacy, Albanese NP, Rouse MJ. Scope of contemporary pharmacy practice: roles, responsibilities, and functions of pharmacists and pharmacy technicians. J Am Pharm Assoc. (C) 2010.10

Abbreviations: BCOP, board-certified oncology pharmacist; BCPS, board-certified pharmacotherapy specialist.

histories that help to elucidate the patient's tolerance of current and previous medications and chemotherapy, and thus also adding to factors considered for selection. ${ }^{11}$

In recent years, some pharmacists have concentrated on pharmacogenomics, the study of genetic factors that contribute to determining drug response or toxicity. ${ }^{12}$ Genome variation for the tumor is due to acquired somatic mutations acquired through the evolution of a cancer. Differences in the tumor genome help to explain varying therapeutic responses to chemotherapy. Examples of this are seen in the selection of epidermal growth-factor receptor $(E G F R)$ drugs like gefitinib, erlotinib, and cetuximab. Identification of somatic mutations in the tyrosine-kinase domain of the EGFR gene will correlate with response. ${ }^{13}$ Variation in the germ-line genome represents interindividual inherited genetic factors. ${ }^{12}$
With identification of genetic marker decisions for optimal drug selection, dose and treatment duration can be tailored to certain patients. Response to chemotherapy has been demonstrated to be a heritable trait dictated by the germ-line genome. Based upon pharmacogenetic testing, the OPS can be involved with selection of a drug that may have a likelihood of a higher response or if specific toxicity risks are higher for one drug over the other. ${ }^{12,13}$

\section{Prescribing, dosing, and transcribing}

Pharmacists play a key role in the complexity of prescribing and transcribing chemotherapy regimens. ${ }^{9}$ Prescribing or ordering is defined as the specific items in a prescription and the logistics of placing the medication order. ${ }^{9}$ Mistakes in prescriptions or in the prescribing process can lead to

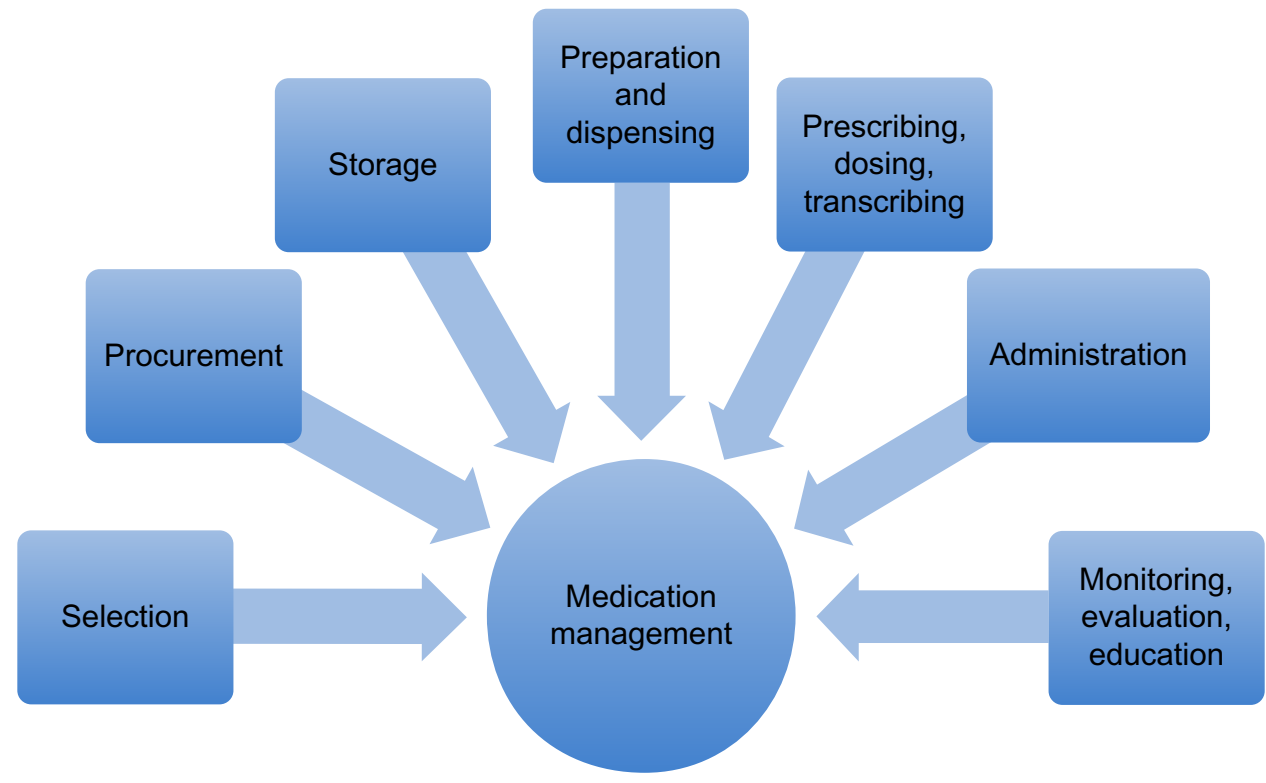

Figure 2 Medication management: seven critical processes from the Joint Commission.

Notes: Data from http://www.jointcommission.org/standards_information/standards.aspx. ${ }^{9}$ 
significant medication errors. General medication errors in hospitals generally range from $2 \%$ to $5 \%$. Chemotherapy error rates have been reported at 3\%-16\%.14,15 Errors in ordering are most common, followed by administration and then dispensing. Missing or improperly written premedication orders prior to chemotherapy and missing treatment parameters could also lead to potentially serious adverse drug reactions. A study investigating the error rate in pediatric acute lymphoblastic leukemia patients found a $10 \%$ oral medication error rate. Errors occurred mainly in prescribing and administration. ${ }^{16-18}$

In 1996, Cohen et al published updates to the 1993 ASHP guidelines to further define safety steps in preventing medication errors in cancer chemotherapy. ${ }^{19}$ These guidelines urged implementation of dose-verification processes, detailed checklists, decrease in the use of verbal orders, and standardizing vocabulary to offset the use of acronyms formed from generic, chemical, and brand names. These updates were in response to a galvanizing medication error that occurred in 1994 at the Dana-Farber Cancer Institute in Boston, MA, USA, when a dose written for cyclophosphamide $4 \mathrm{~g} / \mathrm{m}^{2}$ to be given over 4 days was instead given daily for 4 consecutive days. One patient died and another patient suffered permanent harm..$^{20}$ This error was due to inaccurate interpretation of an unclear order.

Prescribing complex chemotherapy regimens is complicated, with dosing calculations based upon body surface area (BSA) as opposed to set doses and varying administration on consecutive and nonconsecutive days. Other order specifics for infusion times, route, diluents, carrier solutions, container type, and stability are items left to the OPS and/or to pharmacists working directly in the sterile compounding pharmacy area. Standardized chemotherapy order forms have been shown to minimize errors when specific components are included in a standardized order created by OPSs. ${ }^{21}$

Standardized order components include diagnosis, height, most recent weight, BSA calculation, dosage $\left(\mathrm{mg} / \mathrm{m}^{2}\right)$, final calculated dose, start date and time, day of therapy, solution diluent and volume, infusion rate (drips), route (intravenous [IV] push or infusion), duration of infusion, frequency of administration, and total number of scheduled doses. ${ }^{22}$ Documentation of height and weight will help the pharmacist and nurse to double-check calculations of BSA and final dose. ${ }^{22}$

Electronic order entry has allowed for stronger safety measures for oncology chemotherapy orders. The pharmacist can help resolve such system failures as inadequate medication order entry and can help track where failures occur.
OPSs are key players in creating standardized electronic order sets that are linked to clinical laboratory tests and program for medication alerts for interactions and doses that exceed maximum allowable limits.

Practitioner order entry allows for faster and safer pharmacist order verification, and avoids the previous mistakes caused by hand-transcribing a physician order to the medication-administration record. Several hospitals have taken initiatives to implement multidisciplinary failure modes and effects analysis (FMEA) in order to reduce improper dosing, incorrect dosing calculations, assure cumulative dose calculations, and implement checklists for incomplete nursing orders. Even with implementation of computerized physician order entry, the process of FMEA to accompany pharmaceutical care will add to safety measures..$^{23}$

Pharmacists contribute significantly to the dosing portion of the prescribing process. Dosing in elderly and pediatric patients may require consideration of unique factors. Approximately $52 \%$ of cancer patients with newly diagnosed cancers are at least 65 years of age. ${ }^{24} \mathrm{~A}$ natural part of the aging process will affect drug-absorption, distribution, metabolism, and elimination changes. Changes in drug absorption result from decreased splanchnic blood flow and gastrointestinal motility and increased gastric $\mathrm{pH}$. Cardiovascular and gastrointestinal medications can also affect these processes. Physiological changes, such as increase in body fat, decrease in body water and lean body mass, serum proteins, and hemoglobin concentration will affect drug distribution. Hepatic drug-metabolizing enzymes in the cytochrome P450 (CYP) cascade show decreased activity with antineoplastics like the taxanes that undergo hepatic CYP isoenzyme metabolism. Any agent causing inhibition or competition with CYP3A would affect serum levels and increase the potential for toxicity. ${ }^{25}$ All these scenarios would benefit from an OPS assessment for dosage adjustment.

Drug-drug and drug-disease interactions in the elderly may also complicate dosing. Elderly patients have an average of three comorbid conditions and an average of 9.1 medications. Cardiovascular drugs are the most commonly prescribed medications. Paclitaxel and carboplatin were the most commonly used antineoplastic agents. In patients with this combination of medications, dosage adjustments were not made. ${ }^{25-27}$

Aging also affects drug excretion, since the glomerular filtration rate (GFR) decreases. Renally excreted agents like carboplatin will require dose adjustment in declining renal function, since $70 \%$ of the dose is eliminated in urine in the first 24 hours. The combination of decreased excretion and 
drug interactions in the elderly are also potential areas for complications. Common medications utilized in geriatric populations like cyclooxygenase-2 inhibitors may affect elimination of several medications in the antineoplastic category. ${ }^{25}$

Pediatric patients present unique challenges in dosing due to differences in drug disposition. Altered gastrointestinal function, such as $\mathrm{pH}$, motility, and bile production, or enzyme activity will increase or decrease oral drug absorption. Pediatric populations are more vulnerable also from a pharmacokinetic standpoint. Decreased metabolic and renal clearances at birth require drug-dosage adjustments. However, rapid development of organs and tissues affects metabolism and clearance, thus affecting drug serum concentrations. Children 1-10 years old have a larger volume of distribution and may have a higher dose $/ \mathrm{kg}$ requirement. Highly lipid-soluble drugs may have a smaller volume of distribution with resulting higher serum concentrations from a specific dose. All these factors may alter the dosing and require the expertise of a pediatric-trained oncology pharmacist. $^{28}$

As children move through puberty, increased sexhormone production affects both CYP and conjugation metabolic pathways. Adolescent males have increased lean body mass and water, whereas women have increased body fat. These alterations in body composition affect drug distribution. BSA-to-mass ratio changes throughout childhood, with BSA increasing threefold while weight increases 5.5-fold between 2 and 18 years. The question often arises as to when dosing for an adolescent is based upon BSA as opposed to dose $/ \mathrm{kg}$, with GFR adjusted for BSA remaining stable throughout adolescence. Some evidence is available that secretion of drugs is reduced after puberty, thus requiring dose adjustment. ${ }^{27-29}$

Questions are often posed to pharmacists in dosing chemotherapy for both the underweight and overweight patient. Actual body weight is used to calculate BSA for dosing or GFR. However, in the severely underweight patient, numerous alterations exist in drug disposition. Rate and extent of absorption as well as increase in free active drug due to decreased serum proteins may affect distribution. Metabolism and excretion are reduced. Protein and calorie malnutrition also affect a patient's sensitivity to chemotherapy, leading to decreased tumor response. ${ }^{28,31-32}$

In overweight patients, clinicians may hesitate to dose a patient at full body weight. Obesity is defined as 30\%-40\% over ideal body weight or above the 95 th percentile for body mass index. However, reviews of drug disposition in obese patients suggest that serum protein binding, metabolism, and renal function remain unchanged, bringing into question the need for dosage adjustment. ${ }^{33,34}$

Alterations in obese patients' pharmacokinetic profiles include reduced clearance of doxorubicin and methylprednisolone that showed increased acute pharmacological effects. However, reduced clearance has not been correlated with differences in efficacy or toxicity. ${ }^{35,36}$

Pharmacogenomic studies have affected dosing in certain antineoplastics. With the discovery of the thiopurine methyltransferase allele in pediatric acute lymphoblastic leukemia patients, the US Food and Drug Administration (FDA) changed labeling for 6-mercaptopurine to require genetic testing to be performed to determine if a dose decrease is needed. ${ }^{13}$ Pharmacists at the St Jude Children's Research Hospital in Memphis, TN now routinely individualize doses of mercaptopurine based on thiopurine methyltransferase genotype and clinical tolerance. In conjunction with the informatics team, decision-supported alerts are built into computerized orders. An alert fires when a high-risk drug is ordered on a patient with high-risk genotype. ${ }^{37,38}$ The FDA has also changed labeling to recommend pharmacogenetic prescribing for irinotecan in patients with the UGT1A1 (uridine diphosphate glucuronosyltransferase 1 family, polypeptide A1) genetic variation, which may cause patients to suffer from more intense neutropenia or diarrhea. Patients with dihydropyrimidine dehydrogenase genetic variation may need dose adjustments due to increased risk for leucopenia and severe mucositis. ${ }^{13}$

\section{Procurement}

Making a drug available to the patient in and of itself does not help to optimize anticancer therapy, but certainly procurement is a necessary step. The OPS is spending increasing time trying to procure antineoplastics due to manufacturing shortages. A record 320 drug shortages in the US were reported in 2013. Most shortages occur in the injectable dosage form. The FDA indicates that quality issues, such as contamination (43\%), are the most common issues, but other factors, such as manufacturing delays, plant closures, drug discontinuations, and increased demand, add to the shortage. ${ }^{39}$

Drug shortages can cause numerous complications. McBride et al described the most common shortages that occurred 12 months prior to a survey from 243 members of the Hematology/Oncology Pharmacy Association and other organizations. ${ }^{40}$ The most common shortages included leucovorin, liposomal doxorubicin, fluorouracil, and paclitaxel, which may affect patients with ovarian, breast, and 
colon cancer. In $93 \%$ of patients, shortages were reported to have delayed therapy or caused changes in therapy because patients were forced to travel to another institution, receive alternative medications, receive lower doses, or omit medications altogether. Increased cost was reported in $85 \%$ of patients. Reimbursement challenges related to drug shortages were reported in $10 \%$ of patients. Additional costs were also related to increased labor hours, with 1,000 extra hours reported in 34\% of cases. These additional hours spent by pharmacists could be better spent in other areas. Changes in drug therapy due to drug shortages also caused a medication error rate of $6 \%$ and a near-miss error rate of $16 \% .^{39,40} \mathrm{~A}$ study from St Jude Children's Research Hospital linked a shortage of mechlorethamine to a greater risk of relapse for young Hodgkin's lymphoma patients. Relapse in these patients required additional intensive therapy, leaving them at risk for infertility and other treatment-related health problems. ${ }^{41}$

ASHP routinely updates medications in shortage and includes the three drugs listed in McBride et al, as well as carboplatin, cytarabine, dacarbazine doxorubicin, fludarabine, lomustine capsules, mercaptopurine tablets, methotrexate injection and tablets, thiotepa, and vinblastine. ${ }^{42}$ On a daily basis, the OPS role must check on the availability of drugs in shortage and work with oncologists to modify upcoming regimens.

Oral targeted therapy cost can cost more than US $\$ 40,000-\$ 45,000$ per patient. ${ }^{43}$ With this cost, many retail pharmacies may not be willing to expend the budget if they are not guaranteed reimbursement. Specialty pharmacies where OPSs work have become well versed in obtaining preauthorizations and reimbursement for high-cost oral antineoplastics. ${ }^{44}$

\section{Storage}

Proper storage of antineoplastic agents in proper lighting and temperature to maintain chemical integrity is crucial to ensure that antineoplastics maintain their full dose activity. More critical is the issue regarding lookalike/soundalike (LA/SA) medications, improper storage, and how this may impact proper dispensing. Drugs such as vincristine and vinblastine, carboplatin and cisplatin, daunorubicin, doxorubicin, and idarubicin have long been on the Institute for Safe Medication Practices list of drugs with confusing names. ${ }^{45}$ The Joint Commission medication-management standard MM.01.02.01, EP 1 describes the LA/SA standard and details storage and dispensing standards to avoid medication errors. ${ }^{46}$ It cannot be assumed that antineoplastics are separately stored from other medications, especially in small hospital pharmacy departments or a satellite clinic pharmacy. Pharmacists and their support staff must store these LAs/SAs physically apart to ensure proper selection. Other methods to differentiate include special font and size on labeling and applying warning ancillary stickers on the final dispensed drug.

Improper storage placement for antineoplastic agents and nononcology LA/SA medications may also lead to medication errors. For instance, paclitaxel may be confused with Paxil $^{\circledR}$ (GSK, Philadelphia, PA, USA), an antidepressant, or Xenical $^{\circledR}$ (Hoffman-LaRoche, Nutley, NJ, USA) (orlistat), a compound prescribed for weight loss, can be confused with XELODA ${ }^{\circledR}$ (Genentech Inc., South San Francisco, CA, USA) (capecitabine). ${ }^{45}$

\section{Preparation and dispensing}

Reconstitution and preparation of anticancer drugs can be a complex process. Pharmacist-prepared and Pharmacy and Therapeutics Committee-approved standardized charts for dilution quantity, type of carrier solutions and volumes, specific containers (glass, low polyvinyl chloride, and plastic), infusion rates, and expiration dates will help with accurate compounding or preparation. Compounding worksheets that document each cycle of chemotherapy brand used, serial numbers (in case of recall), calculating cumulative doses of certain drugs, cycle number of chemotherapy and dates, along with space for technicians and pharmacists to double-check help to record a patient's course of therapy over time. OPSs can evaluate the use of closed-system transfer devices like PhaSeal $^{\circledR 47}$ (Becton Dickinson, Franklin Lakes, NJ, USA) or ChemoClave $^{\circledR 48}$ (ChemoClave Hospira, Inc., Lake Forest, IL, USA) may help to prime the chemotherapy safely for use by the administrator. OPSs are also responsible for making sure that multiple chemotherapy agents are not placed into biological safety cabinets simultaneously, so as to avoid injection of the wrong drug into the carrier solution. ${ }^{9}$

Preparation areas in outpatient, inpatient, and satellite pharmacy spaces may be small and not allow for appropriate ventilation. OPSs should have a role in assuring facility compliance with regulatory standards for biological safety, such as for vertical hoods or safety cabinets. This ensures safety for the technician and pharmacist, and also assures a sterile product. ${ }^{49}$

For research protocols, the OPS, nurse study coordinator, and investigational new drug pharmacist can work together to share information on compounding of medications, since there can be limited data on stability, precipitation, and administration. ${ }^{10}$ The pharmacist's role in dispensing is to assure that the final drug product is legally labeled and 
contains all ancillary notification either on the label or stickers for administration, storage, and destruction. Examples include "Hazardous drug", "Refrigerate", or "Return to pharmacy for destruction". 9

Dispensing also includes appropriate delivery, and OPSs are key in outlining policies and procedures for appropriate transport. For instance, fragile and costly biologicals should not be transported in tube-delivery systems to avoid destruction of the fragile protein carriers and thus negation of drug efficacy. OPS expertise is critical in creating policies and procedures that detail containment in spills or leak situations and ensuring proper disposal of IV, oral, or topical dosage forms. ${ }^{9}$

\section{Administration}

Administration remains a nursing mainstay, especially for parenteral drugs. Nurses often ask for information on compatibilities with multiple infusion lines, scheduling and sequencing, infusion rates, volume issues to prime IV lines properly, manipulating concentrations in fluid restriction, or even changing the type of carrier solutions in cases of metabolic abnormalities. In cases of delayed infusion time or delay in delivery, discussion on infusion rates to make up infusion time needs to occur, especially where short time frames are given for drug stability. Discussion on viable IV access, whether via centrally placed infusion catheters as opposed to peripherally placed lines, may help to avoid extravasation, especially in cases of past extravasation. Providing supportive care in cases of extravasation antidotes and protocols is also a vital role for the OPS. ${ }^{50}$

Dispensing and administration errors involving parenteral medications in pediatric populations can cause devastating outcomes. Due to the small volume of IV vincristine and the IV push administration, inadvertent intrathecal administration of vincristine was first reported in $1968 .{ }^{51,52}$ Intrathecal administration of vincristine can cause acute central neurotoxicity and lethal outcomes. From 1968 to 2006, 55 cases of inadvertent intrathecal administration have been reported worldwide. Physician/nurse and pharmacy error occurred in $69 \%$ of cases, pharmacy error $19 \%$, and physician/nurse error $12 \% .{ }^{53}$ In cases where all three disciplines were involved, inadequate communication was cited as the most common cause. In pharmacy-only errors, mislabeling of syringes was the main cause. Physician and nurse failures occurred due to lack of checking of labeling or to check physician orders. In 2005, The Joint Commission released a "sentinel event alert" in order to prevent vincristine administration errors. Standards detail steps to avoid past mistakes. More recently, vincristine has been prepared in IV piggyback so that volumes will prohibit intrathecal administration. ${ }^{54}$

Nurses and OPSs must work together to prepare a patient properly to receive chemotherapy. OPSs can prepare orders for prehydration and forced-diuresis protocols in high-dose chemotherapy regimens. ${ }^{53}$ Suggestions to utilize such chemoprotective agents as high-dose ifosfamide with mesna, amifostine, and dexrazoxane are often made by pharmacists with regard to appropriate dosing, administration times, and sequence. OPSs can assure that protective steroid eyedrops are ordered to protect against conjunctivitis caused by high-dose cytarabine. ${ }^{50}$ Infusion of monoclonal antibody drugs, such as rituximab and trastuzumab, may require prophylaxis with such medications as corticosteroids, antihistamine, and acetaminophen to avoid anaphylactic or allergic reactions. ${ }^{53}$

Administration preparedness also includes prophylaxis in supportive care areas, such as preventing nausea and vomiting. Historic use of the dopamine antagonists metoclopramide and prochlorperazine utilized the skills of the pharmacist for prophylaxis against extrapyramidal side effects. With the advent of the 5- $\mathrm{HT}_{3}$-antagonist class of antiemetics, OPSs collaborate with physicians to establish prophylaxis protocols that match levels of regimen emetogenicity. OPSs have shown that algorithms and treatment guidelines have shown cost reductions and improved control of chemotherapy-induced nausea and vomiting (CINV). ${ }^{55}$ Most recent reports show how pharmacist interventions help to reduce costs of antiemetics by $16 \%$ in outpatient clinics. ${ }^{57,58}$ The release of such neurokinin-1 receptor antagonists as aprepitant add to the CINV regimens, especially in patients receiving multiday chemotherapy and cisplatin therapy. The correct utilization of aprepitant has been demonstrated in solid and hematological tumors, for transplant by pharmacists as a supportive regimen. ${ }^{59-64}$

In delayed $\mathrm{CINV}$, clinicians tend to overprescribe aprepitant and serotonin antagonists and underprescribe corticosteroids. Physicians also tend to underprescribe antiemetics in patients receiving low-emetogenic-potential regimens. ${ }^{65-67}$ OPSs can help to choose correct medications other than the serotonin-receptor antagonists based upon protocols agreed upon between oncologists and OPSs.

Administration challenges also occur with oral dosage forms. The placement of OPSs in inpatient and outpatient care areas and into specialty pharmacies has increased their role in administration. The US National Cancer Institute reports that $25 \%$ of the 400 antineoplastic agents in the FDA pipeline are planned to be oral medications. ${ }^{68,69}$ 
Table 3 Targeted therapies for cancer

\begin{tabular}{|c|c|c|c|}
\hline Drug & $\begin{array}{l}\text { Trade } \\
\text { name }\end{array}$ & $\begin{array}{l}\text { Antigen } \\
\text { target }\end{array}$ & Indication \\
\hline \multicolumn{4}{|l|}{ Monoclonal antibody } \\
\hline Alemtuzumab & Campath & CD52 & B-cell CLL \\
\hline Bevacizumab & Avastin & VEGF & MM \\
\hline Bortezomib & Velcade & $N F-\kappa B$ & MCR \\
\hline Cetuximab & Erbitux & EGFR & MCR \\
\hline Denosumab & Xgeva & RANKL & GCT \\
\hline Dabrafenib & Tafinlar & BRAF & MM \\
\hline $\begin{array}{l}\text { Gemtuzumab } \\
\text { ozogamicin }\end{array}$ & Mylotarg & CD33 & AML \\
\hline Ipilimumab & Yervoy & CTLA4 & MCR \\
\hline Ibritumomab tiuxetan & Zevalin & CD20 & MM \\
\hline Panitumumab & Vectibix & CD20 & $\mathrm{NHL}$ \\
\hline Pertuzumab & Perjeta & HER2 & $\mathrm{BCA}$ \\
\hline Ponatinib & Iclusig & BCR-ABL & CLL \\
\hline Rituximab & Rituxan & EGFR & NHL, CLL \\
\hline Tositumomab & Bexxar & CD20 & $\mathrm{NHL}$ \\
\hline Trametinib & Mekinist & MEKI and 2 & MM \\
\hline Trastuzumab & Herceptin & HER2 & MBC \\
\hline Ofatumumab & Arzerra & CD20 & CLL \\
\hline \multicolumn{4}{|l|}{ Tyrosine-kinase inhibitors } \\
\hline Afatinib & Gilotrif & $\begin{array}{l}\text { EGFR, HER2, } \\
\text { HER4 }\end{array}$ & NSCLC \\
\hline Bosutinib & Bosulif & Bcr-Abl & CML \\
\hline Erlotinib & Tarceva & EGFR & NSCLC \\
\hline Gefitinib & Iressa & EGFR & NSCLC \\
\hline Imatinib & Gleevec & Bcr-Abl & $\mathrm{CML}$ \\
\hline \multicolumn{4}{|l|}{ Multikinase inhibitors } \\
\hline Cabozantinib & Cometriq & VEGF, RET & MTC \\
\hline Sorafenib & Nexavar & VEGFR, PDGFR & $\mathrm{HCC}, \mathrm{RCC}$ \\
\hline Sunitinib & Sutent & VEGFR, KIT & $\begin{array}{l}\text { RCC, GIST, } \\
\text { PNET }\end{array}$ \\
\hline Pazopanib & Votrient & TKI & Kidney, STC \\
\hline Regorafenib & Stivarga & VEGFR & CRC \\
\hline
\end{tabular}

Abbreviations: AML, acute myelogenous leukemia; $B C A$, breast cancer; $C L L$, chronic lymphocytic leukemia; GCT, giant-cell tumor; NHL, non-Hodgkin's lymphoma; NSCLC, non-small-cell lung cancer; CRC, colorectal cancer; MCR, metastatic colorectal; CML, chronic myeloid leukemia; MBC, metastatic breast cancer; MM, metastatic melanoma; HCC, hepatocellular cancer; RCC, renal cell carcinoma; GIST, gastrointestinal stromal tumor; PNET, pancreatic neuroendocrine tumor; CD52, CAMPATH-I antigen or cluster of differentiation 52; VEGF, vascular endothelial growth factor; NK-KB, nuclear factor kappa-light-chain-enhancer of activated B-cells; EGFR, epidermal growth factor receptor; RANKL, receptor activator of nuclear factor kappa-B ligand; BRAF, proto-oncogene B-Raf; CD33, siglet 3; CTLA4, cytotoxic T-lymphocyte antigen 4; CD20, activated-glycosylated phosphoprotein; HER2, human epidermal growth factor receptor 2; BCR-ABL, the $\mathrm{Ph}$ chromosome defective mutated gene; EGFR, epidermal growth factor receptor; MEKI and 2, mitogen-activated protein kinase kinase; HER4, human epidermal growth factor receptor 4; RET, rearranged during transfection; PDGFR, platelet derived growth factor receptor; KIT, receptor tyrosine kinase and a type of tumor marker; TKI, tyrosine kinase inhibitor; STC, soft-tissue sarcoma; MTC, medullary thyroid cancer.

Notes: Data taken from. ${ }^{68}$

Since 2011, 26 new oral chemotherapy agents have been FDA-approved, with the majority as targeted therapies, as shown in Table 3. In a survey of 42 US national cancer centers, few had systems established for monitoring and managing risks linked with oral chemotherapy. ${ }^{70,71}$
The key issue in oral therapy is drug adherence, formerly known as drug compliance. General rates of medication adherence vary from $20 \%$ to $100 \% .^{72}$ Assumptions are made that adherence is not an issue in oncology patients, since cancer is a life-threatening disease. In women receiving oral cyclophosphamide and prednisone for breast cancer, self-reported compliance rates of $57 \%$ were reported. Bonadonna and Valagussa established an association between missed chemotherapy doses and inferior disease-free survival. ${ }^{73}$ Several studies indicate that higher survival rates occur with patient concordance with oral chemotherapy regimens. ${ }^{74,75}$ In a curable hematological malignancy, a 44\% compliance rate was measured via assay, even with patient education. ${ }^{76,77}$

Additional issues arise with prolonged or long-term oral therapy. A patient's perception of risks associated with disease, benefits from therapy, and the nature and severity of side effects affects long-term adherence. Social and psychological factors, such as social support, financial resources, complexity of treatment, and the patient's general belief about health and wellness warrant consideration. ${ }^{75,78,79}$

Pharmacists can employ various tools, such as a diary, to help with patient adherence and confirm adherence with pill counts and verification with refill histories. ${ }^{78}$ Patients must understand the importance of communicating missed

Table 4 Oncology pharmacist role in oral-targeted therapy

- Preauthorization of prescription

- Third-party reimbursement expertise

- Patient education on safety for handling and disposal

- Medication administration

- Taken with or without food

- Crushability

- Address issues for dissolution of capsule contents

- Allergic reaction - hives and itching, hypotension - prophylaxis with histamine antagonist (diphenhydramine)

- Flu-like symptoms - chills, fatigue, fever, muscle aches, and pain (acetaminophen, corticosteroid)

- Nausea - antiemetics

- Adverse side effect management

○ Diarrhea

- Skin rashes

- More serious side effects

- Infusion reactions - shortness of breath, hypotension

- Hematological - white blood cells and platelets

- Cardiac - congestive heart failure

- Dermatological - sores and rashes, mucositis

- Bleeding

- Monitor adherence

- Pill counts

- Verification with refill 
doses to their caregivers. Pharmacist phone calls will also help to establish rapport. Table 4 summarizes the oncology pharmacist's role in managing oral therapies.

\section{Monitoring and evaluation}

The last major medication-management process of monitoring and evaluating drug therapy has long been an area that OPSs have helped in to optimize anticancer drug therapy. By joining clinical rounds and suggesting clinical interventions at the bedside, the OPSs can effect faster change. ${ }^{11}$

As mentioned in the "Prescribing, dosing, and transcribing" section, medication errors may also cause adverse drug reactions. Pharmacists and nurses have demonstrated the ability to respectively avert potential adverse drug reactions by $50 \%$ and $40 \%$. Occasionally even the patient may play a role in circumventing adverse drug reactions. ${ }^{80}$

During chemotherapy administration, the OPS can monitor the patient's ability to tolerate hydration regimens, electrolyte abnormalities, possible tumor lysis syndrome, control of nausea, vomiting, and other acute side effects via patient interview and routine monitoring of chemicals and vital signs. OPSs on patient-care floors or in IV compounding areas in pharmacies can chart or log cumulative doses for drugs like anthracycline for cardiac toxicity, with recommendations for multigated acquisition scan (MUGA), the nuclear image test that measures ejection fraction or perform pulmonary function tests for such pulmonary toxic drugs as bleomycin..$^{55}$

Gastrointestinal toxicities, such as diarrhea and mucositis, can be prevalent with antineoplastic antimetabolites like fluorouracil and irinotecan. All grades of diarrhea have been reported as high as $82 \%$. OPSs can help with creating protocols to prevent or treat irinotecan-induced diarrhea with practice guidelines for assessment, dietary management, and pharmacological management with loperamide, octreotide, and antibiotics when necessary. ${ }^{81,82}$ Mucositis occurs alongside neutropenia, and OPSs can help with ordering prophylaxis or prompt ordering of various mouth rinses or local anesthetics to promote good oral hygiene by minimizing oral fungal infection and helping to minimize pain. ${ }^{11}$

Patients may need IV support or nutritional support during or between cycles of chemotherapy, due to nausea/vomiting, prolonged mucositis, enteritis, diarrhea, significant weight loss, cancer cachexia, and dysgeusia. Multidisciplinary care teams rely on pharmacists for a comprehensive initial assessment. ${ }^{83}$ Weight loss may be a diagnostic factor in certain cancers, and cachexia is a hallmark of cancer patients, exhibited by early satiety, weight loss, and asthenia. ${ }^{28,84-86}$
Poor nutrition can impact the patient's ability to tolerate recommended doses of chemotherapy and ability to recover from chemotherapy side effects. It is essential for the health care team to utilize the OPS to maximize the nutrition status of the patient in order for optimum antineoplastic dose administration. Working with dietitians to maintain adequate nitrogen balance and any issues of substrate metabolism, such as hyperglycemia, lipid metabolism, and maintaining a neutral or positive nitrogen balance, requires a team effort from pharmacist, dietitian and nurses. ${ }^{28,87}$

Common side effects of chemotherapy are hematological, such as anemia, thrombocytopenia, and neutropenia. OPSs can monitor absolute neutrophil counts and platelet and hemoglobin levels to assure blood parameters are within acceptable limits for the next cycle of chemotherapy. ${ }^{55}$

Pharmacists have played a key role in antibiotic selection, dosing, and pharmacokinetic monitoring, especially in the case of the febrile neutropenic patient. ${ }^{88}$ Interventions have demonstrated a shortened number of total days due to focused attention by pharmacists. ${ }^{89}$ Studies have demonstrated beforeand-after effects from pharmacist-led antibiotic-stewardship programs. Significant decline in usage rates for inappropriate antibiotic use have been demonstrated, as well as for average duration of therapy. In the absence of pharmacists from the stewardship team, Clostridium difficile infection increased more than threefold. ${ }^{90}$

OPS assessment and evaluation of a patient's ability to tolerate oral medications and diet can expedite the conversion of IV antibiotics to the oral formulation, especially in patients where high-risk neutropenia is downgraded with fever defervescence, neutrophil recovery, and resolution of mucositis..$^{91,92}$

As mentioned earlier, genetic variation in UGT1A1 is associated with irinotecan-induced neutropenia. Pharmacist monitoring of these patients would help avert potential infectious complications. ${ }^{13}$

The majority of US states allow pharmacists to administer vaccinations..$^{93}$ OPSs can interview patients before initiation of chemotherapy to assess the need for influenza, diphtheria, tetanus, and pneumococcal vaccines. Patient education for medication compliance and finishing complete courses of antibiotic therapy to decrease resistance patterns is also key for pharmacists. ${ }^{94-97}$

OPSs can be very active in managing dermatological or cutaneous adverse effects caused by chemotherapy. Creating and updating extravasation protocols and guidelines is one of the first duties of any OPS. ${ }^{11}$ Other dermatological issues can range from skin changes, such as nail reactions, 
hyperpigmentation, photosensitivity, rashes, and limb/ palmar-plantar erythematous reactions. In the taxane class of drugs, grade 3 toxicity can occur in $19 \%$ of patients. ${ }^{55}$ Pharmacists can provide a systematic approach to managing cutaneous reactions with utilization of warm or cold compresses, topical and/or systemic antibiotics, and topical and/ or systemic corticosteroids. ${ }^{98}$

In general, OPSs can play a vital role in monitoring and evaluating drug therapy. ${ }^{99}$ Broadfield described a rating sheet for four common toxicities - nausea, vomiting, diarrhea, and stomatitis - that was administered to patients by oncology nurses. Patients rated their toxicity on the day of chemotherapy, and blood work was documented. Pharmacists in the clinic would utilize these ratings to validate appropriate timing for dispensing of medications and also solve any necessary interventions. This helped to minimize waiting time for patients as well. A second phase of their program for toxicity monitoring included development of agent-specific toxicity scales. Toxicity profiles were described with recommendations for clinical monitoring. The standardized format ensured that key toxicities were addressed, and any toxicity could be extracted for outcome indicators. ${ }^{100}$ Protocols and standardized order forms help by having a recognizable framework to improve outcomes, minimize adverse effects, and provide patient education.

\section{Patient education}

Patient education is essential to empower the patient in their own care, and educated patients have also played a role in catching medication errors themselves, especially if receiving repeated cycles. Patients have detected omitted premedications, wrong infusion intervals, leaking infusions, and incorrect doses, especially of oral medications. ${ }^{101-102}$ In an outpatient setting, OPSs can counsel new chemotherapy patients with a review of all the patient's medications, including prescriptions, over-the-counter, vitamins, alternative therapy, and herbal products, for drug-chemotherapy interactions, drug-drug interactions, duplicate therapy, and potential side effects. Counseling services can also include patient expectations at clinic visits, education on adverse effects, compliance with supportive care medications, and any lifestyle modifications, such as contraception, diet, and fall-prevention precautions. ${ }^{103}$

Patient education is paramount to support successful oral treatment. ${ }^{104,105}$ Oncology pharmacists in ambulatory care settings and specialty retail pharmacies can play a prime role to help reduce outpatient and inpatient hospital visits and decrease administration and home-care costs. ${ }^{106}$
Acute therapy issues, such as adverse effects and complex dosing regimens, and administration issues, such as taking drugs with or without food, will affect absorption and thus efficacy. Whether or not a capsule can be taken apart or contents dissolved, or if a tablet like gefitinib can be crushed is imperative information for patients who have difficulty swallowing. ${ }^{107}$

Patients may also need education on proper handling and storage of oral agents. Medication-information sheets, nonabsorbable gloves, and hazardous waste disposal containers should accompany an outpatient oral chemotherapy prescription. Patients should be advised to avoid crushing or manipulating the dosage form without consulting an oncology pharmacist. Patient caregivers in the home or other nontraditional settings, such as long-term care facilities, should transfer the medication from the prescription container directly into the medication cup. Accidentally dropping a pill or capsule would require gloves or a paper towel to retrieve before being disposed of in a hazardous waste receptacle. ${ }^{108}$

Oral agents have added side effect profiles to the usual side effect profile. Palmar-plantar erythrodysesthesia syndrome can include acral erythema, hand-foot syndrome, and hand-foot skin reactions, and can occur with such multikinase inhibitors as sorafenib and sunitinib. ${ }^{55}$ Epidermal growth-factor receptor tyrosine-kinase inhibitors have a $75 \%-90 \%$ incidence of papulopustular rash and maculopapular acneiform rash. The incidence increases when these drugs are concomitantly administered with a monoclonal antibody. Hand-foot syndrome can occur with capecitabine, a side effect that can progress to blistering and desquamation. Skincare education, such as decreasing exposure to hot water, friction, and trauma in early therapy, avoidance of tight fitting shoes, and rigorous exercise are helpful prevention tactics, as are moisturizing with appropriate pressure in grade 0 and educating patients on application of creams and gels if these rashes progress to grades of $1-3 .{ }^{109,110}$

Rereleased medications like thalidomide are available through restricted-access programs to avoid the repeat mishaps of birth defects and fetal death. Pharmacist intervention is required in assuring proper contraception, especially since thalidomide is present in semen. Pharmacists can clarify hormonal versus nonhormonal contraception choices depending upon the tumor type, and advise patients not to donate blood or sperm during therapy and for 4 weeks after cessation of therapy. ${ }^{53}$

The establishment of a pharmacist-developed anticoagulation clinic and the importance of education has been 
documented. ${ }^{111}$ Pharmacists and pharmacy technician-led education programs in breast cancer clinics have demonstrated better patient understanding of chemotherapysupport medications and led to significant dose reductions, reductions in the number of chemotherapy delays, and the amount of repeat dispensing of chemotherapy support medications. ${ }^{112}$

The link between thrombosis and malignancy is well documented in cancer patients who have concomitant venous thromboembolism, having increased mortality over noncancer patients. ${ }^{113}$ Cancer patients also experience increased complications and recurrence with chronic anticoagulation with warfarin. ${ }^{114}$

The use of chemotherapy is a known risk factor for the development of venous thromboembolism. ${ }^{115}$ Certain chemotherapy agents, such as fluorouracil and capecitabine, are known to interact specifically with warfarin. ${ }^{116}$ Endocrine therapy, mainly tamoxifen, has been associated with an increased risk of thrombosis. ${ }^{117}$ In a review of over 5,000 breast cancer patients seen in pharmacist-managed anticoagulation services demonstrated, $65.6 \%$ of patients were within therapeutic range as opposed to $56.7 \%$ of patients who were not seen by pharmacists. The incidence of major bleeds in this study was $2.1 \%$, similar to nononcology patients entered in randomized trials $(0.9 \%-1.8 \%) .{ }^{118}$ Other trials in oncology patients have reported major bleeds at $12 \%-13 \%{ }^{119,120}$

In a survey performed by McKee et al of patients in outpatient settings, $86 \%$ felt it was "absolutely necessary" to discuss their initial treatment with a pharmacist, and 76\% of surveyed patients requested pharmacy follow-up at future visits. Patients were willing to pay for pharmacy counseling services (83\%), with $28.9 \%$ willing to pay between USD $\$ 10$ and \$20 and $19.7 \%$ willing to spend more than \$20 on pharmacy services. ${ }^{103}$

\section{Conclusion}

\section{Vital members}

\section{of the interdisciplinary team}

As members of interdisciplinary team members, oncology pharmacy specialists offer a variety of services related to the seven processes of medication management, such as procurement and storage, and contributing to selection, prescribing, dosing, monitoring, evaluation and education. ${ }^{10,121,122}$ Pharmaceutical care can take the form of improving management of supportive care, enhancing patient education, improving efficiency in the chemotherapy infusion center, and creating disease-specific treatment guidelines. ${ }^{99}$ OPSs contribute heavily to ensuring the safety of antineoplastic medications in order for them to be utilized to their fullest therapeutic potential. Involvement in gathering and follow-up of patientspecific information and treatment adds strength to assessment and prioritization of care. ${ }^{121}$ In many US states and other countries, OPSs can enter into collaborative practice agreements to help manage drug therapy of patients under physician supervision. These types of practices can occur in hospitals, ambulatory care centers, and specialty pharmacies, and can range from the traditional dispensing of drugs to an independent role of running support-care clinics for managing side effects. ${ }^{121}$

\section{Disclosure}

The author reports no conflicts of interest in this work.

\section{References}

1. Morris CR, Hickman MJ. Medical oncology pharmacy: a new role for the clinical pharmacist. Am J Pharm Educ. 1977;41:278-280.

2. [No authors listed]. ASHP technical assistance bulletin on handling cytotoxic and hazardous drugs. Am J Hosp Pharm. 1990;47:1033-1049.

3. American Society of Hospital Pharmacists. ASHP statement on pharmaceutical care. Am J Hosp Pharm. 1993;50:1720-1723.

4. [No authors listed]. ASHP guidelines on preventing medication errors in hospitals. Am J Hosp Pharm. 1993;50:305-314.

5. ASHP Council on Professional Affairs. ASHP guidelines on preventing medication errors with antineoplastic agents. Am J Health-Syst Pharm. 2002;59:1648-1668.

6. Board of Pharmacy Specialies. [webpage on the Internet] http://www. bpsweb.org/about/history.cfm. Accessed January 25, 2014.

7. American College of Clinical Pharmacy. Frequently asked questions about board certification. Available from: https://www.accp.com/ careers/boardfaq.aspx. Accessed September 1, 2013.

8. Cancer.Net. ASCO expert corner: the role of the oncology pharmacist. Available from: http://www.cancer.net/all-about-cancer/ cancernet-feature-articles/expert-information-asco/asco-expert-cornerrole-oncology-pharmacist. Accessed November 1, 2013.

9. Joint Commission. About our standards. Available from: http://www. jointcommission.org/standards_information/standards.aspx. Accessed October 22, 2013

10. Council on Credentialing in Pharmacy, Albanese NP, Rouse MJ. Scope of contemporary pharmacy practice: roles, responsibilities, and functions of pharmacists and pharmacy technicians. J Am Pharm Assoc (2003). 2010;50:e35-e69.

11. Wong WM, Ignoffo RF. If there are expert systems and dose checks, why do we still need the clinical pharmacist? Pharm Pract Manage $Q$ 1996;16:50-58.

12. O'Donnell PH, Ratain MJ. Germline pharmacogenomics in oncology: decoding the patient for targeting therapy. Mol Oncol. 2012;6:251-259.

13. Moen El, Godley LA, Zhang W. Dolan ME. Pharmacogenomics of chemotherapeutic susceptibility and toxicity. Genome Med. $2012 ; 4: 90$

14. Taylor JA, Winter L, Geyer LJ, Hawkins DS. Oral outpatient chemotherapy medication errors in children with acute lymphoblastic leukemia. Cancer. 2006;107;1400-1406.

15. Beyzarov E. High rate of drug errors seen in pediatric oncology. Pharm Pract News. 2006;33:10.

16. Bates DW, Boyle DL, Vander Vliet MB, Schneider J, Leape L. Relationship between medication errors and adverse drug events. J Gen Intern Med. 1995:10:199-205 
17. Gandhi TK, Weingart SN, Seger AC, et al. Outpatient prescribing errors and the impact of computerized prescribing. J Gen Intern Med. 2005;20:837-841.

18. Goldspiel BR, DeChristoforo R, Daniels CE. A continuous-improvement approach for reducing the number of chemotherapy-related medication errors. J Health Syst Pharm. 2000;57 Suppl 4: S4-S9.

19. Cohen MR, Anderson RW, Attilio RM, Green L, Muller RJ, Pruemer JM. Preventing medication errors in cancer chemotherapy. Am J Health Syst Pharm. 1996;53:737-746.

20. Brink S. Tragedy at Dana-Farber: Betsy Lehman's shocking death is still roiling the medical community. 1995. Available from: http:// health.usnews.com/usnews/health/articles/950724/archive_032493. htm. Accessed December 28, 2013.

21. Pastel DA, Fay P, Lee D. Effect of implementing a cancer chemotherapy order form on prescribing habits for parenteral antineoplastics. Hosp Pharm. 1993;28:1192-1195.

22. Serrano-Fabiá A, Albert-Marí A, Almenar-Cubells D, JiménezTorres NV, Multidisciplinary system for detecting medication errors in antineoplastic chemotherapy. J Oncol Pharm Pract. 2010;16: $105-112$.

23. Kim GR, Chen AR, Arceci RJ, et al. Error reduction in pediatric chemotherapy: computerized order entry and failure modes and effects analysis. Arch Pediatr Adolesc Med. 2006;160:495-498.

24. Surveillance, Epidemiology, and End Results Program. SEER cancer statistics review (CSR) 1975-2010. 2013. Available from: http://seer. cancer.gov/csr/1975_2010. Accessed November 1, 2013.

25. Sokol KC, Knudsen JF, Li MM. Polypharmacy in older oncology patients and the need for an interdisciplinary approach to side-effect management. J Clin Pharm Ther. 2007;32:169-175.

26. Safran D, Neuman P, Schoen C, et al. Prescription drug coverage and seniors: findings from a 2003 national survey. Health Aff (Millwood). 2005; Suppl Web Exclusives: W5-152-W5-166.

27. Gurwitz JH, Field TS, Harrold LR, et al. Incidence and preventability of adverse drug events among older persons in the ambulatory setting. JAMA. 2003;289:1107-1116.

28. Ladas EJ, Sacks N, Meacham L, et al. A multidisciplinary review of nutrition considerations in the pediatric oncology population: a perspective from children's oncology group. Nutr Clin Pract. 2005;20:377-393.

29. Kearns GL, Abdel-Rahman SM, Alander SW, Blowey DL, Leeder JS, Kauffman RE. Developmental pharmacology - drug disposition, action and therapy in infants and children. $N$ Engl J Med. 2003;349: $1157-1167$.

30. Carr R, Ensom M. Drug disposition and therapy in adolescence: the effects of puberty. J Pediatr Pharmacol Ther. 2003;8:86-96.

31. Krishnaswamy K. Drug metabolism and pharmacokinetics in malnourished children. Clin Pharmacokinet. 1989;17 Suppl 1:68-88.

32. Murray DJ, Riva L, Poplack DG. Impact of nutrition on pharmacokinetics of anti-neoplastic agents. Int J Cancer Suppl. 1998;11:48-51.

33. Blouin RA, Warren GW. Pharmacokinetic considerations in obesity. J Pharm Sci. 1999;88:1-7.

34. Cheymol G. Effects of obesity on pharmacokinetics implications for drug therapy. Clin Pharmacokinet. 2000;39:215-231.

35. Rodvold KA, Rushing DA, Tewksbury DA. Doxorubicin clearance in the obese. J Clin Oncol. 1998;6:1321-1327.

36. Dunn TE, Ludwig EA, Slaughter RL, Camara DS, Jusko WJ. Pharmacokinetics and pharmacodynamics of methylprednisolone in obesity. Clin Pharmacol Ther. 1991;49:536-549.

37. Relling MV. Pharmacogenomics of adverse effects of anti-leukemic agents in children. J Pediatr Pharmacol Ther. 2012;17(1):7-11.

38. Crews KR, Cross SJ, McCormick JN, Baker DK, et al. Development and implementation of a pharmacist-managed clinical pharmacogenetics service. Am J Health Syst Pharm. 2011;68:143-150.

39. US Food and Drug Administration. A review of FDA's approach to medical product shortages. 2011. Available from: http://www.fda.gov/ AboutFDA/ReportsManualsForms/Reports/ucm275051.htm. Accessed October 22, 2013.
40. McBride A, Holle LM, Westerndorf C, et al. National survey on the effect of oncology drug shortages on cancer care. Am J Health Syst Pharm. 2013;70:609-617.

41. Metzger ML, Billett A, Link M. Impact of drug shortages on children with cancer - the example of mechlorethamine. New Engl J Med. 2012;367:2461-2463.

42. American Society of Health-System Pharmacists. Dacarbazine injection. 2013. http://www.ashp.org/DrugShortages/Current/Bulletin. aspx?id=949. Accessed October 19, 2013.

43. Plevin DM, Ward HM, Ward MB, Sorich MJ, McKinnon RA. Pharmacist's role in targeted cancer therapy in Australia and implications for pharmacy education. Am J Pharm Educ. 2010;74:168

44. Jones S. Pharmacy practice issues with targeted therapy for lung cancer. Am J Health-Syst Pharm. 2003;60:11-15.

45. Joint Commission. Look-alike/sound-alike drug list. 2011. Available from: http://www.jointcommission.org/LASA. Accessed October 22, 2013.

46. Institute for Safe Medication Practices. ISMP's list of confused drug names. 2011. Available from: http://www.ismp.org/Tools/ confuseddrugnames.pdf. Accessed October 22, 2013.

47. Becton Dickinson. BD PhaSeal closed system transfer device. 2013. Available from: http://www.BD.com/pharmacy/phaseal. Accessed November 2, 2013.

48. ICU Medical [website on the Internet]. Available from: http://www. icumedical.com. Accessed November 2, 2013.

49. Tanaka GJ, Fujiwara J, Kramer H, Bredt AB, Wortmann M. Justification and implementation of an oncology pharmacist practitioner in an HMO setting. Hosp Pharm. 1985;20:568-574.

50. Hepler CD, Strand LM. Opportunities and responsibilities in pharmaceutical care. Am J Hosp Pharm. 1990;47:533-543.

51. Schochet SS Jr, Lampert PW, Earle KM. Neuronal changes induced by intrathecal vincristine sulfate. Neuropathol Exp Neurol. 1968;27:645-658.

52. Bain PG, Lantos PL, Djurovic V, West I. Intrathecal vincristine: a fatal chemotherapeutic error with devastating central nervous system effects. J Neurol. 1991;238:230-234.

53. Lagman JL, Tigue CC, Trifilio SM, et al. Inadvertent intrathecal administration of vincristine. Community Oncol. 2007;4:45-46.

54. Joint Commission [website on the Internet]. Available from: http://www. jointcommission.org. Accessed November 2, 2013.

55. Lexicomp [website on the Internet]. Available from: http://www.lexicomp.com. Accessed December 27, 2013.

56. Berard CM, Mahoney CD. Cost-reducing treatment algorithms for antineoplastic drug-induced nausea and vomiting. Am J Health Syst Pharm. 1995;52:1879-1885.

57. Iihara, H, Ishihara M, Matsuura K, et al. Pharmacists contribute to the improved efficiency of medical practices in the outpatient cancer chemotherapy clinic. J Eval Clin Prac. 2010;18:753-760.

58. Basch E, Prestrud AA, Hesketh PJ, et al. Antiemetics: American Society of Clinical Oncology clinical practice guideline update. J Clin Oncol. 2011;29:4189-4198.

59. DeWit R, Herstedt J, Rapoport B, et al. Addition of the oral NK1 antagonist aprepitant to standard antiemetics provides protection against nausea and vomiting during multiple cycles of cisplatin based chemotherapy. J Clin Oncol. 2003;21:4105-4111.

60. Warr DG, Hesketh PJ, Gralla RJ, et al. Efficacy and tolerability of aprepitant for the prevention of chemotherapy-induced nausea and vomiting in patients with breast cancer after moderately emetogenic chemotherapy. J Clin Oncol. 2005;23:2822-2830.

61. Hesketh PJ, Grunberg SM, Gralla RJ, et al. The oral neurokinin-1 antagonist aprepitant for the prevention of chemotherapy-induced nausea and vomiting: a multinational, randomized, double-blind, placebo-controlled trial in patients receiving high-dose cisplatin - the Aprepitant Protocol 052 Study Group. J Clin Oncol. 2003;21:4112-4119.

62. Bubalo JS, Cherala G, McCune JS, Munar MY, Tse S, Maziarz R. Aprepitant pharmacokinetics and assessing the impact of aprepitant on cyclophosphamide metabolism in cancer patients undergoing hematopoietic stem cell transplantation. J Clin Pharmacol. 2012;52:586-594. 
63. Paul B, Trovato JA, Thompson J, Badros AZ, Goloubeva O. Efficacy of aprepitant in patients receiving high-dose chemotherapy with hematopoietic stem cell support. J Oncol Pharm Pract. 2010;16: 45-51.

64. Uchida M, Ikesue H, Kato K, et al. Antiemetic effectiveness and safety of aprepitant in patients with hematologic malignancy receiving multiday chemotherapy. Am J Health Syst Pharm. 2013;70:343-349.

65. National Comprehensive Cancer Network. Clinical practice guideline in oncology - antiemesis v1.2012. Available from: www.NCCN org/professionals/physician-gls/f_guidelines.asp\#antiemesisNCCNclinicalpracticeguidelinesinoncology. Accessed November 5, 2013.

66. Hesketh PJ, Warr DG, Street JC, Carides AD. Differential time course of action of 5-HT3 and NK1 receptor antagonists when used with highly and moderately emetogenic chemotherapy (HEC and MEC). Support Care Cancer. 2011;19:1297-1302.

67. Chan AC, Shih V, Chew L. Evolving roles of oncology pharmacists in Singapore: a survey on prescribing patterns of antiemetics for chemotherapy induced nausea and vomiting (CINV) at a cancer centre. J Oncol Pharm Pract. 2008;14:23-29.

68. US Food and Drug Administration. Drugs: hematology/oncology (cancer) approvals and safety notifications. Available from: http:// www.fda.gov/drugs/informationondrugs/approveddrugs/ucm 279174 htm\#updates. Accessed October 30, 2013.

69. Weingart SN, Spencer J, Buia S, et al. Medication safety of five oral chemotherapies: a proactive risk assessment. J Oncol Pract. 2011;7:2-6.

70. Backes K, Griesbach S, Wilhelm S, Plank G. Identification of drug therapy opportunities with oral chemotherapy. 2013. Available from: http://www.specialtypharmacytimes.com/publications/specialtypharmacy-times/2013/May_June-2013/Identification-of-Drug-TherapyOpportunities-with-Oral-Chemotherapy. Accessed December 27, 2013.

71. Weingart SN, Flug J, Brouillard D, et al. Oral chemotherapy safety practices at US cancer centres: questionnaire survey. BMJ. 2007;334:407.

72. Partridge AH, Avorn J, Wang PS, Winer EP. Adherence to therapy with oral antineoplastic agents. J Natl Cancer Inst. 2002;94:652-662.

73. Bonadonna G, Valagussa P. Dose response effect of adjuvant chemotherapy in breast cancer. $N$ Engl J Med. 1981;304:10-15.

74. Porzsolt F, Meuret G, Kreuser ED, et al. Compliance of physicians and patients with a consensus protocol for treatment of advanced breast cancer. J Cancer Res Clin Oncol. 1994;115:564-570.

75. Spiegel D. Psychosocial aspects of breast cancer treatment. Semin Oncol. 1997;24:S136-S147.

76. Lebovits AH, Strain JJ, Schleifer SJ, Tanaka JS, Bhardwaj S, Messe MR. Patient noncompliance with self-administered chemotherapy. Cancer. 1990;65:17-22.

77. Levine AM, Richardson JL, Marks G, et al. Compliance with oral drug therapy in patients with hematological malignancy. J Clin Oncol. 1987;5:1469-1476.

78. Viele CS. Managing oral chemotherapy: the healthcare practitioner's role. Am J Health Syst Pharm. 2007;64:S25-S32.

79. Love RR, Cameron L, Connell BL, Leventhal H. Symptoms associated with tamoxifen treatment in postmenopausal women. Arch Intern Med. 1991;151:1842-1847.

80. Gandhi TK, Bartel SV, Shulman LN, et al. Medication safety in the ambulatory chemotherapy setting. Cancer. 2005;104:2477-2483.

81. Cherny NI. Evaluation and management of treatment-related diarrhea in patients with advanced cancer: a review. J Pain Symptom Manage. 2008;36:413-423.

82. Maroun JA, Anthony LB, Blais N. Prevention and management of chemotherapy-induced diarrhea in patients with colorectal cancer: a consensus statement by the Canadian Working Group on chemotherapy-induced diarrhea. Curr Oncol. 2007;14: 13-20.

83. ASPEN Board of Directors and the Clinical Guidelines Task Forces. Guidelines for the use of parenteral and enteral nutrition in adult and pediatric patients. JPEN J Parenter Enteral Nutr. 2002;26:1SA-1388SA.
84. American Society for Parenteral and Enteral Nutrition. Clinical nutrition critical care guidelines released [press release]. Silver Spring (MD): ASPEN; April 27, 2009. Available from: http://www. nutritioncare.org/News/Clinical_Nutrition_Critical_Care_Guidelines_Released. Accessed November 3, 2013.

85. Mauer AM, Burgess JB, Donaldson SS, et al. Special nutritional needs of children with malignancies: a review. J PEN J Parenter Enteral Nutr. 1990;14:315-324.

86. Langer CJ, Hoffman JP, Ottery FD. Clinical significance of weight loss in cancer patients: rationale for the use of anabolic agents in the treatment of cancer-related cachexia. Nutrition. 2001;17:S1-S20.

87. Dunn R, Stettler N, Mascarenhas M. Refeeding syndrome in hospitalized pediatric patients. Nutr Clin Pract. 2003;18: 327-332

88. Briceland LL, Lomaestro BM, Griswold MW, Lesar TS. Interventions in antimicrobial therapy made by infectious diseases clinical pharmacy specialists. J Infect Dis Pharmacother. 1999;(4):319-331.

89. Masiá M, Matoses C, Padilla S, et al. Limited efficacy of a nonrestricted intervention on antimicrobial prescription of commonly used antibiotics in the hospital setting: results of a randomized controlled trial. Eur J Clin Microbiol Infect Dis. 2008;27:597-605.

90. Cappelletty D, Jacobs D. Evaluating the impact of a pharmacist's absence from an antimicrobial stewardship team. Am J Health Syst Pharm. 2013;70:1065-1069.

91. Dunn K, O’Reilly A, Silke B, Rogers T, Bergin C. Implementing a pharmacist-led sequential antimicrobial therapy strategy: a controlled before-and-after study. Int J Clin Pharm. 2011;33:208-214.

92. Oosterheert JJ, Bonten MJ, Schneider MM, et al. Effectiveness of early switch from intravenous to oral antibiotics in severe community acquired pneumonia: multicenter, randomized trial. $B M J$. 2006;333:1193-1198.

93. Centers for Disease Control and Prevention. Prevention strategies for seasonal influenza in healthcare settings. 2013. Available from: http:// www.cdc.gov/flu/professionals/infectioncontrol/healthcaresettings. htm. Accessed October 28, 2013.

94. Dellit TH, Owens Rc, McGowan JE Jr, et al. Infectious Diseases Society of America and the Society for Healthcare Epidemiology of America guidelines for developing an institutional program to enhance antimicrobial stewardship. Clin Infect Dis. 2007;44:159-177.

95. Drew RH, White RW, MacDougall C, Hermsen ED, Owens RC. Insights from the Society of Infectious Diseases pharmacists on antimicrobial stewardship guidelines from the Infectious Diseases Society of America and the Society for Healthcare Epidemiology of America. Pharmacotherapy. 2009;29:593-607.

96. MacDougall C, Polk RE. Antimicrobial stewardship programs in health care systems. Clin Microbiol Rev. 2005;18:638-656.

97. [No authors listed]. ASHP statement on the pharmacist's role in antimicrobial stewardship and infection prevention and control. Am J Health Syst Pharm. 2010;67:575-577.

98. Chew L, Chuen VS. Cutaneous reaction associated with weekly docetaxel administration. J Oncol Pharm Pract. 2009;15:29-34.

99. Valgus JM, Faso A, Gregory KM, et al. Integration of a clinical pharmacist into the hematology-oncology clinics at an academic medical center. Am J Health Syst Pharm. 2011;68:613-619.

100. Broadfield L. Pharmaceutical care in oncology pharmacy practice: a method for using outcome indicators. J Oncol Pharm Pract. 1995;1:9-14.

101. Schwappach DL, Wernli M. Medication errors in chemotherapy: incidence, types and involvement of patients in prevention. A review of the literature. Eur J Cancer Care (Engl). 2010;19:285-292.

102. Shah S, Dowell J, Greene S. Evaluation of clinical pharmacy services in a hematology/oncology outpatient setting. Ann Pharmacother. 2006;40:1527-1533.

103. McKee M, Frei BL, Garcia A, Fike D, Soefje SA. Impact of clinical pharmacy services on patients in an outpatient chemotherapy academic clinic. J Oncol Pharm Pract. 2010;17:387-394.

104. Aisner J. Overview of the changing paradigm in cancer treatment: oral chemotherapy. Am J Health Syst Pharm. 2007;64:S4-S7. 
105. Hartigan K. Patient education: the cornerstone of successful oral chemotherapy treatment. Clin J Oncol Nurs. 2003;7:21-24.

106. Pituskin E, Fairchild A, Dutka J, et al. Multidisciplinary team contributions within a dedicated outpatient palliative radiotherapy clinic: a prospective descriptive study. Int J Radiat Oncol Biol Phys. 2010;78: 527-532.

107. Steiber D. Oncology? The brass ring? 2013. Available from: http:// www.specialtypharmacytimes.com/publications/specialty-pharmacytimes/2013/May_June-2013/Oncology-The-Brass-Ring. Accessed December 27, 2013.

108. Jones KL, Garnett C, Gauthier M, Boster B, Espirito JL, Michaud LB. Clinical outcomes of a pharmacist-managed anticoagulation service for breast cancer patients. J Oncol Pharm Pract. 2011;18: 122-127.

109. Bartel SB. Safe practices and financial considerations in using oral chemotherapeutic agents. Am J Health Syst Pharm. 2007;64:S8-S14.

110. Lacouture ME, Anaadkat MJ, Bensadoun RJ, et al. Clinical practice guidelines for the prevention and treatment of EGFR inhibitor-associated dermatologic toxicities. Support Care Cancer. 2011;19:1079-1095.

111. Wood LS, Lemont H, Jatoi A, et al. Practical considerations in the management of hand-foot skin reaction caused by multikinase inhibitors. Community Oncol. 2010;7:23-29.

112. Panglilinan JA. Venous thromboembolism in patients with cancer: an overview for pharmacists using a case-based approach. J Pharm Pract. 2010;23:294-302.

113. Read H, Ladds S, Rhodes B, Brown D, Portlock J. The impact of supplementary medication review and counseling service within the oncology outpatient setting. Br J Cancer. 2007;96:744-751.

114. Sørensen HT, Mellemkjaer L, Olsen JH, Baron JA. Prognosis of cancers associated with venous thromboembolism. $N$ Engl J Med. 2000;343:1846-1850.
115. Rose AJ, Sharmann JP, Ozonoff A, Henault LE, Hylek EM. Effectiveness of warfarin among patients with cancer. J Gen Intern Med. 2007;22:997-1002.

116. Heit JA, Silverstein MD, Mohn DN, Petterson TM, O’Fallon WM, Melton LJ 3rd. Risk factors for deep vein thrombosis and pulmonary embolism: a population based case-control study. Arch Intern Med. 2000;160:809-815.

117. Saif MW. An adverse reaction between warfarin and fluoropyrimidines revisited. Clin Colorectal Cancer. 2005;5:175-180.

118. Deitcher SR, Gomes MP. The risk of venous thromboembolic disease associated with adjuvant hormone therapy for breast carcinoma: a systematic review. Cancer. 2004;101:439-449.

119. Prandoni P, Lensing AW, Piccioli A, et al. Recurrent venous thromboembolism and bleeding complications during anticoagulant treatment in patients with cancer and venous thrombosis. Blood. 2002;100: 3484-3488.

120. Hutten BA, Prins MH, Gent M, Ginsberg J. Incidence of recurrent thromboembolic and bleeding complications among patients with venous thromboembolism in relation to both malignancy and achieved international normalized ratio: a retrospective analysis. J Clin Oncol. 2000;18:3078-3083.

121. Hutchison LC, Castleberry A. The pharmacist's new role as part of an interdisciplinary care team. J Am Soc Aging. 2012;35:62-68.

122. Liekweg A, Westfeld M, Jaehde U. From oncology pharmacy to pharmaceutical care: new contributions to multidisciplinary cancer care. Support Care Cancer. 2004;12:73-79.
Integrated Pharmacy Research and Practice

\section{Publish your work in this journal}

Integrated Pharmacy Research and Practice is an international, peer-reviewed, open access, online journal, publishing original research, reports, reviews and commentaries on all areas of academic and professional pharmacy practice. This journal aims to represent the academic output of pharmacists and pharmacy practice with particular focus on integrated care. All papers are carefully peer reviewed

\section{Dovepress}

to ensure the highest standards as well as ensuring that we are informing and stimulating pharmaceutical professionals. The manuscript management system is completely online and includes a very quick and fair peer-review system, which is all easy to use. Visit http://www.dovepress.com/testimonials.php to read real quotes from published authors. 